\title{
Does Low Dose Steroids Confer Benefits in Sepsis and Septic Shock?
}

\author{
Nazim $\mathrm{SN}^{\mathrm{a}}$
}

\begin{abstract}
Over the last 40 years, the use of corticosteroids in sepsis and septic shock has evolved from the initial use of high-dose, short-duration steroid therapy in the 1980s, to the recent recommendation of using low-dose longerduration steroid therapy in refractory septic shock patients. A recent, prospective, open-label, randomized, controlled pilot trial of patients in four adult intensive care units in London-teaching hospitals showed that hydrocortisone decreased vasopressin requirements, reduced the duration and reduced the required dose, when used together in the treatment of septic shock, but it did not alter plasma vasopressin levels. The optimal dosing of hydrocortisone, about $300 \mathrm{mg} /$ day, was agreed uniformly. As this dose also provides sufficient mineralocorticoid effects, the additional use of mineralocorticoid is not needed. Despite the fact that severely ill septic shock patients are given treatment, according to the current guidelines, the survival benefit is unproven. The benefits of steroids on less severely ill septic patients are still questionable. More research is highly recommended to successfully identify the benefits of steroids in septic shock.
\end{abstract}

Key words: benefit, low dose steroid, sepsis, septic shock

(BIRDEM Med J 2017; 7(1): 48-49)

\section{Introduction}

Sepsis is a life-threatening condition that develops when the body responds to infection in a way that damages its own tissues and organs. Sepsis may progress to septic shock, multi-organ dysfunction syndrome (MODS) and death, especially if not treated early. Although some of the therapies in sepsis carry little controversies, the benefits of using the exogenous corticosteroids in septic shock are still unclear. Since the first randomized controlled trial (RCT) on the corticosteroid for severe sepsis, it has been used broadly by clinicians worldwide. The broad acceptance of steroids in septic shock is likely due to its prompt reversal of life-threatening conditions, such as septic shock and respiratory failure. Given the crucial role of steroids in septic shock, which is evident in many trials, it is important to evaluate the rationale for using corticosteroids and also whom it will confer benefits and at what stage and when.

\section{Author Informations}

a. Dr. Sarah Nehrina Nazim, MSc in Critical Care Medicine, The Queen Mary University of London.

Address of correspondence: Dr. Sarah Nehrina Nazim, MSc in Critical Care Medicine, The Queen Mary University of London, Email: dr.sarahnazim@gmail.com

Received: August 1, 2016

Accepted: November 30, 2016

\section{Definition of sepsis and septic shock}

The original definitions of sepsis, severe sepsis, and septic shock are more than 20 years old. A new definition, 'SEPSIS-3', has now been published which is produced by the European Society of Intensive Care Medicine (ESICM) and Society of Critical Care Medicine (SCCM) Sepsis Redefinitions Task Force.

\section{Sepsis-3 definition}

Sepsis is characterized by a life-threatening organ dysfunction caused by a dysregulated host response to an infection. ${ }^{1}$

\section{Sepsis - clinical criteria}

Organ dysfunction is defined as an acute change in total SOFA scores by $\geq 2$ points according to the infection. The baseline SOFA score can be assumed to be zero in patients not known to have any pre-existing organ dysfunction. It is assumed that a patient with a SOFA score of two or more along with an infection roughly has $10 \%$ chance of mortality. Patients with suspected infections that are prone to have a prolonged ICU care or about to die in the hospital can be promptly identified at the bedside with qSOFA (quick SOFA), i.e.

- $\quad$ Systolic blood pressure $\leq 100 \mathrm{~mm} \mathrm{Hg}$ 
- $\quad$ Altered mental status (any Glasgow Coma Scale $<15$ )

- $\quad$ Respiratory rate $>22 / \mathrm{min}$

Septic shock: It is a subset of sepsis in which the underlying circulatory and cellular/metabolic abnormalities are altered and associated with increased mortality. ${ }^{1}$

Septic shock - clinical criteria: Septic patients with persisting hypotension, requiring vasopressors to maintain mean arterial pressure (MAP) $65 \mathrm{~mm} \mathrm{Hg}$, and also have a serum lactate level $>2 \mathrm{mmol} / \mathrm{L}(18 \mathrm{mg} / \mathrm{dL})$, despite adequate volume resuscitation. With these criteria, hospital mortality is in the abundance of $40 \%$.

\section{Pathogenesis of sepsis}

In the pathogensisis of sepsis there remain role of organism and body immune response, microcirculatory alterations and role of Inflammatory Cytokines. ${ }^{2-4}$ Cellular response to sepsis is given in figure 1 .

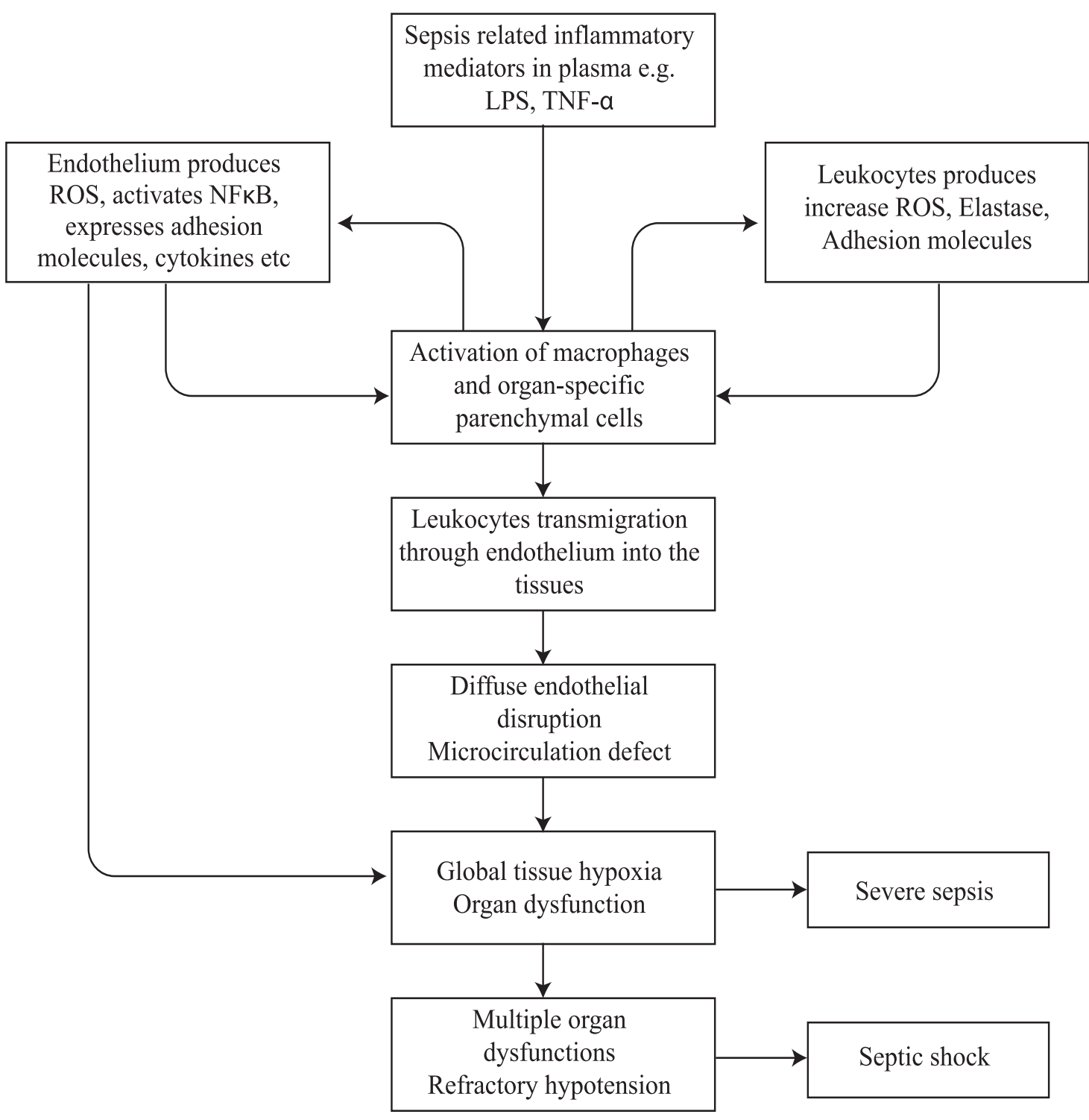

Figure-1: Schematic diagram of cellular responses to sepsis (Adopted from Bersten and Soni, n.d.) (Cepinskas and Wilson, 2008) 


\section{Effect of critical illness on the HPA axis}

Stress can at the first supersede standard regulatory mechanism and the diurnal rhythm of cortisol secretion. There is an increased release of CRH and ACTH and a reduced negative feedback effect of cortisol; resulting in hypercortisolism in critical illness. Increased cortisol exerts its effects to maintain homeostasis during stress. A rise in serum cortisol level is observed in the critically ill patients, and this increased level correlates with the severity of the infection. ${ }^{5}$

In contrast 'ACTH, cortisol dissociation' phenomenon can be seen in critically ill patients. In this phenomenon, the ACTH concentration falls below the normal control values, whereas plasma cortisol levels remain elevated. ${ }^{6}$ Increased cortisol levels were thought to be a result of reduced cortisol breakdown in critical illnesses. ${ }^{7}$ Chronic exposure to IL-6, IL-1â and TNFá may result in decreased ACTH and CRH production. ${ }^{5}$

It is also demonstrated that the level of corticosteroidbinding globulin (CBG) during critical illness falls rapidly and results in an expanded level of free corticosteroids. ${ }^{8}$ The $\mathrm{CBG}$ gets cleaved by the enzyme neutrophil elastase, at the site of inflammation, consequently decreasing the affinity for cortisol and liberates free cortisol at the site of inflammation.

Despite the fact that a sustained activation of the HPA axis is vital in critical illnesses, the mechanism of its dysfunctional activation is poorly understood. When dysfunctional HPA axis activation is unable to cover the requirements of cortisol for survival, and the plasma cortisol levels are higher than normal, the condition has been labeled as 'relative adrenal insufficiency' (RAI). ${ }^{9}$

Critical illness related cortical insufficiency (CRICI) Sepsis-associated adrenal insufficiency develops due to insufficient cortisol secretion from the adrenal glands. An inadequate secretion of cortisol impairs the inflammatory response and is unable to meet the metabolic demand. ${ }^{10}$ This effect was recently termed as critical illness-related corticosteroid insufficiency (CIRCI). Insufficient corticosteroid secretion downregulates the inflammatory transcription factors, which is a common manifestation of CIRCI. ${ }^{11}$ Similar to type 2 diabetes CIRCI may arise due to corticosteroid tissue resistance along with HPA axis suppression. The transcriptional activity of the GR-á is inhibited by the inflammatory products such as TNFá, which interferes with its interaction with p160 type nuclear receptor coactivators.

\section{Factors related to cortical insufficiency}

It is evident that multiple mechanisms are responsible for causing GC resistance during sepsis, including reduced numbers of GRs, reduced affinity of GR to GC, altered nuclear receptor co-activators and the, increased conversion of cortisol to cortisone. ${ }^{12}$

During sepsis, a subset of patients may have a structural defect of the adrenal or pituitary gland, inflammation of the endocrine tissues or may experience anatomically damaged gland which impairs cortisol synthesis. Certain drugs may inhibit the enzymatic steps of cortisol synthesis, (e.g. etomidate, ketoconazole), whereas some may increase cortisol metabolism (e.g. Phenytoin, Phenobarbital). ${ }^{13}$ Patients receiving these drugs may indeed experience altered cortisol synthesis. Several proinflammatory cytokines released during sepsis focus on the reversible dysfunction of HPA axis.It has also been demonstrated that positive blood culture and infection with Gram-negative organisms predisposes to adrenal insufficiency.

Diagnostic assessment of adrenal function in septic shock patients

\section{ACTH stimulation test}

The dose of ACTH - to assess the integrity of the HPA axis is a subject of discussion.

Supraphysiological dose (high dose): It is debatable because of the supra-physiological doses of ACTH, i.e., $250 \mu \mathrm{g}$ ) may overcome any ACTH resistance, and ACTH stimulation tests may be poorly reproducible in septic shock patients. ${ }^{12}$ Interestingly, many trials have considered the ACTH stimulation test as the best biochemical marker to identify a decreased adrenal function in critically ill patients. In one study of 102 patients with septic shock, $22.5 \%$ were detected with adrenal failure by using ACTH stimulation test. ${ }^{14}$ In this study, the authors evaluated multiple approaches to identify the adrenal failure in septic patients. Finally, they concluded that the standard ACTH stimulation test with $250 \mu \mathrm{g} \mathrm{ACTH}$ and an incremental increase of d" $9 \mu \mathrm{g} /$ deal in total cortisol after 60 minutes was a better option to identify adrenal failure whenever free cortisol measurement was not available. ${ }^{14}$

Low dose: Another trial comparing a low dose $(1 \mu \mathrm{g})$ corticotropin stimulation test with the standard $(250 \mu \mathrm{g})$ test for the diagnosis of RAI concluded that septic patients who were nonresponders to low dose $(1 \mu \mathrm{g})$ ACTH tests had a worse survival rate.

Random cortisol: A random cortisol (serum free cortisol plus the protein bound fraction) of $<10 \mu \mathrm{g} / \mathrm{deal}$ has been 
recommended as a diagnostic tool for CIRCI. ${ }^{12}$ However, the total serum cortisol concentration depends on the rate of its synthesis from the adrenal gland, on its secretion, distribution, binding and also on its elimination. It is not possible to assess the cortisol secretion rate by a single random cortisol measurement since it secretes in a pulsatile manner.

Plasma free cortisol: In critical illness results in a decreased plasma protein, which consequently results in a relative increase in free cortisol level. The plasma free cortisol assessment is not available promptly and cannot be incorporated into everyday clinical practice.

Therefore, the current recommendation does not support the provocative stimulation test of the HPA axis with ACTH to identify the shock patients who should receive glucocorticoids. ${ }^{15}$ Rather, the guideline recommended using a random cortisol level of $<18 \mu \mathrm{g} / \mathrm{dl}$ to diagnose the adrenal failure in the septic patients. ${ }^{16}$

The latest recommendation of the ACCM Consensus Task Force is to diagnose adrenal insufficiency in critical illnesses by a delta cortisol (the change in serum cortisol after $250 \mu \mathrm{g}$ ACTH) of $<9 \mu \mathrm{g} / \mathrm{dl}$ or a random cortisol of $<10 \mu \mathrm{g} / \mathrm{dl} .{ }^{12}$

\section{Rationale for using steroids in sepsis Effects of corticosteroids on circulation}

Studies had demonstrated that corticosteroids may be beneficial in critically ill shock patients who require prolonged vasopressors and ventilator supports. ${ }^{17}$ It's been suggested that corticosteroids reestablish adequate blood volumes by means of sodium and water retention by binding with the renal mineralocorticoid receptors. ${ }^{18}$ Steroids potentiate catecholamine effects by increasing the expression of adrenergic receptors in the vessel wall ${ }^{19}$ and thus, help to restore the systemic vascular resistance.

However, corticosteroids also effectively hinder the release of inducible nitric oxide synthase. results in lengthening the activation of $\mathrm{NO}$ and NFêB following sepsis. ${ }^{20}$

The SSC (Sepsis surviving campaign) decided level $2 \mathrm{C}$ proposal to consider exogenous steroids in patients with the septic shock that are refractory to fluids and vasopressors. ${ }^{16}$

\section{Metabolic effects}

During stress, corticosteroids increase the blood glucose level by stimulating hepatic gluconeogenesis and glycogenolysis ${ }^{13}$, besides, they also increase the delivery of glucose to cells. Moreover, they induce peripheral insulin resistance, thus inhibit the cellular uptake of glucose. Corticosteroids increase the secretion of other hormones, such as glucagon, and adrenaline. Corticosteroids potentiate the release of free fatty acid from adipose tissue. They also interfere with protein synthesis and potentiate proteolysis in muscles. Such metabolic effects of corticosteroids help to supply energy and substrates to the cell during stress eventually repair cells from stress injury. ${ }^{21}$

\section{Anti-inflammatory and immunosuppressive action}

The GR (glucocorticoid receptor)mediates the antiinflammatory and immunosuppressive effects of corticosteroids. Glucocorticoids enter the cell and bind with their particular GRs GRá to form the activated receptor GC-GRá complexes and translocate into the nucleus. The anti-inflammatory and immunosuppressive effects of GC can be divided into two processes, Transactivation and Transrepression. In the case of Transactivation, GC-GR complex binds with their DNAbinding sites, GRE, which are located in the promoter region of target genes. ${ }^{22}$ Thus, it activates the transcription of anti-inflammatory proteins, such as Annexin-1, IêB. Activation of Annexin-1 reduces the liberation of free arachidonic acids and also prostaglandins, from membrane phospholipids. Glucocorticoids also dephosphorylate MAP Kinase Phosphatase-1 (MKP-1) and inactivate c-Jun N-terminal Kinase, which thus inhibits the transcription of inflammatory genes. ${ }^{23}$

On the other hand, Transrepression leads to the repressed expression of pro-inflammatory and immune-regulatory proteins such as IL1, TNFá, and prostaglandins. In this process, the hormone receptor complex interacts with transcription factors such as NFêB (a nuclear transcription factor), which thus inhibits proinflammatory cytokine (for example, TNF- $\alpha$, IL-1, IL6) production. ${ }^{22,24}$ NFêB is also responsible for transcription of cyclooxygenase-2, which is needed to produce prostaglandins. Thus, by inhibiting NFêB, glucocorticoids interfere with prostaglandin synthesis.

Increased amounts of inflammatory markers correlate with the disease severity and worse outcome. ${ }^{19}$

Corticosteroids are known to reduce the eosinophil and basophil cell counts, whereas the Neutrophil counts increases. It is also suggested that glucocorticoids interfere with the traffic of leukocytes into the sites of inflammation, by repressing the expression of adhesion molecules such as ELAM-1 and ICAM-1.Glucocorticoids exert their anti-inflammatory effects by acting on distinct targets through various mechanisms. 


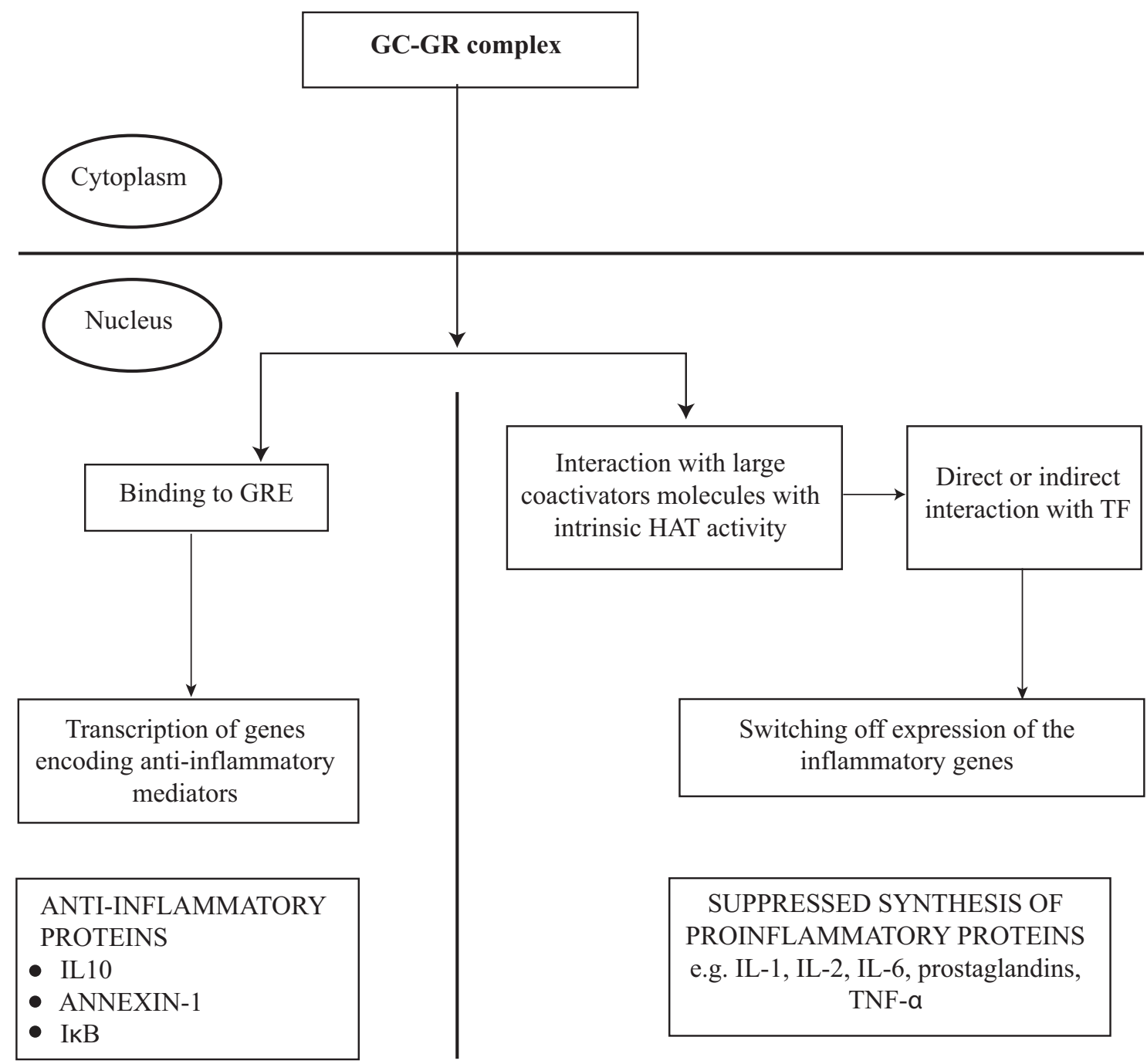

Figure 2: Mechanisms underlying the Glucocorticoid-induced antagonism of inflammation (Adopted from strehl \& Buttgereit, 2013).

\section{Adapting the Surviving Sepsis Guideline}

Evidence based guideline: To improve the standard care rendered towards the septic and septic shock patients, the Society of critical care medicine(SCCM), Europian societ of internal medicine(ESICM) and the International Sepsis Forum launched the Surviving Sepsis Campaign (SSC). It was introduced in the year 2002 at the ESICM Annual meeting and progressed through three phases- thus, it has published three editions of evidence-based guidelines. These guidelines are an ongoing collective process. They are expected to incorporate the best available evidence from clinical trials into practice guidelines. ${ }^{25}$ Most of the medical community received the guidelines with much enthusiasm.

\section{Aims and objectives of the SSC guideline}

A group of experts came together to give guidelines to the bedside clinicians with an aim to improve the outcome of severe sepsis and septic shock. The guidelines were put together in bundles. A performance improvement program was introduced based on these bundles. The bundles were considered as necessary, change drivers in clinical practice established on the quality of available data. However, the recommendations of these guidelines are not meant to replace the clinician's decision-making capability. 


\section{Quality assessment of SSC}

In 2004, experts utilized modified Delphi methodology for grading recommendations based on a 2001 publication supported by the International Sepsis Forum. In following editions of the guidelines, the quality of evidence was evaluated by predefined Grades of Recommendation, Assessment, Development and Evaluation (GRADE) criteria. ${ }^{16}$ This system classifies the quality of evidence as high (Grade A), moderate (Grade B), low (Grade C) or very low (Grade D).

The GRADE system classifies recommendations as strong (Grade 1) or weak (Grade 2)

\section{Evolving recommendation on using steroids in sepsis}

The uncertainties regarding the use of steroids are circulating with the 1.dosing and 2.timing of administrations, and also relating to the 3.diagnosis of adrenal dysfunction. Earlier the guideline suggested the use of steroids in patients who required vasopressor therapy to maintain adequate blood pressure, despite adequate fluid resuscitation (grade C). One large RCT supported this recommendation; in which $200 \mathrm{mg}$ of hydrocortisone was associated with a reduction of approximately $10 \%$ in the mortality rate in 300 septic patients. However, the mortality benefit was limited to patients with an impaired cortisol response to ACTH administration(i.e .non responders).

This manual proposed the use of hydrocortisone treatment in patients who were poorly responsive to fluid resuscitation and vasopressors therapy (Grade 2C). ${ }^{16}$ The conflicting results had led clinical equipoise and the joint decision by members of the SSC to advise using low-dose steroids in septic shock patients who were both fluid and vasopressors-unresponsive.

The most up-to-date recommendations were published in 2013. These guidelines were largely identical to the 2008 guidelines regarding the use of steroids. The recent guideline did not offer the use of Hydrocortisone for adult septic shock patients if adequate fluid resuscitation and Vasopressor therapy could restore hemodynamic stability (level C recommendation). If the hemodynamic stability was unachievable, the guideline suggested using 200mg IV Hydrocortisone per day. But the dose of vasopressor to be used before corticosteroid was not specified in any of the guidelines. But the 2012 guideline did not mention anything about the use of dexamethasone or fludrocortisone-rather, it focused only on the use of hydrocortisone.

Evolving recommendation towards dosing and the discontinuation of steroids in septic patients

300mg vs 200 mg/day: The guidelines of 2004 and 2008 recommended doses of hydrocortisone, not more than $300 \mathrm{mg}$ a day to be used in septic patients. It was characterized as a grade A recommendation in 2004 and grade $1 \mathrm{~A}$ recommendation in 2008, the strongest possible recommendation using the GRADE system of SSC. ${ }^{16}$ The current guideline-recommended a hydrocortisone dose of $200 \mathrm{mg} /$ day (weak recommendation) in refractory septic shock, Continuous infusion vs intermittent dosing : In the recent guideline of 2013, they recommended using continuous infusion rather than repetitive bolus injections (the previous guideline recommended hydrocortisone for 7 days in 2 to 3 divided doses or by continuous infusion. ${ }^{26}$ The rationale was based on the fact that several randomized trials on the use of lowdose hydrocortisone in septic shock revealed a significant risk of hyperglycemia and hypernatremia. ${ }^{27}$ Another small prospective trial demonstrated high blood sugar with the repetitive bolus administration of hydrocortisone, which was not detectable during continuous infusion.

Tappering or abrupt cessation of steroid: There is certainly no comparative study between a fixed duration and clinically guided regimen or between tapering and the abrupt cessation of steroids. According to the 2004 guidelines, the corticosteroid dose should be tapered off at the end of therapy. ${ }^{26}$ RCTs and metanalysis results predict the optimal duration of steroid therapy it is still uncertain. It is not even clear whether tapering off the dose of steroids may affect the outcome. ${ }^{16}$

\section{A single center experience of interpretation and translation of Surviving Sepsis Guideline into bedside practice $^{27}$}

A total of on 1185 septic patients' from 2009 to 2011 were assigned to this study. The investigators concluded 
that the most common threshold for prescribing corticosteroids was the presence of two or more vasopressors which occurred in 99 patients (64\%). Notably, 91 out of 99 had at least one vasopressors infusing at high-doses when corticosteroid was ordered. Of the patients who survived to have their hydrocortisone dose changed, $57 \%$ (60 of 106) had their corticosteroid tapered, whereas $43 \%$ were abruptly discontinued. The decision of tapering or discontinuation of corticosteroids was independent of whether patients still required vasopressors.

To determine whether the provider's attitude towards prescribing corticosteroids for septic shock matched the actual behavior, a survey was developed to ascertain provider beliefs relevant to this issue. $77 \%(n=27)$ of Intensivists responded that they always consider or almost always consider steroids in septic shock patients when they were poorly responsive to vasopressor therapy. The majority of respondents replied that they would prescribe corticosteroids more than 48 hours after the diagnosis of septic shock..
The dosing regimens commonly used for hydrocortisone were $50 \mathrm{mg}$ every 6 hours and $100 \mathrm{mg}$ every eight hours. Nearly half of the providers felt that the vasopressors had to be off for $>24$ hours before changing the corticosteroids dose regimen, whereas $30 \%$ were willing to taper or discontinue corticosteroids despite the patient still being on vasopressors.

These results highlight in part, the difficulty in translating published guidelines to bedside practice

The study concluded that significant variability exists for prescribing corticosteroids in septic shock, with the most common interpretation of 'poorly responsive' to fluid and vasopressor therapy.

Treatment strategies with corticosteroids and related controversies

History of steroids in the treatment of sepsis and septic shock

Table I. Summaries of the meta-analyses of the two large studies (Adopted from legering \& Nevgebor 1995)

\begin{tabular}{|c|c|c|c|c|}
\hline Author & Drug & Dose & Duration & $\begin{array}{c}\text { Risk ratio } \\
\text { (95\% confidence } \\
\text { interval) }\end{array}$ \\
\hline Bone et al 1987 & MP & $30 \mathrm{mg} / \mathrm{kg}$ & 24 hours & $1.35(0.98-1.84)$ \\
\hline Sprung et al 1984 & MP & $30 \mathrm{mg} / \mathrm{kg}$ & $1-2$ doses & $1.11(0.74-1.67)$ \\
\hline $\begin{array}{l}\text { Veterans } \\
\text { Administration } \\
1987\end{array}$ & MP & $\begin{array}{c}30 \mathrm{mg} / \mathrm{kg} \text { followed } \\
\text { by } 5 \mathrm{mg} / \mathrm{kg}\end{array}$ & 9hours & $0.95(0.57-1.8)$ \\
\hline $\begin{array}{l}\text { Luce et al } \\
1988\end{array}$ & MP & $\begin{array}{c}30 \mathrm{mg} / \mathrm{kg} \\
(\times 4)\end{array}$ & $24 \mathrm{hrs}$ & $1.07(0.72-1.6)$ \\
\hline Lucas et al 1984 & $\mathrm{DM}$ & $6 \mathrm{mg} / \mathrm{kg}$ & $48 \mathrm{hrs}$ & $1.09(0.36-3.27)$ \\
\hline $\begin{array}{l}\text { Thompson et al } \\
1976\end{array}$ & MP & $30 \mathrm{mg} / \mathrm{kg}$ & Up to $4 \mathrm{hrs}$ in $24 \mathrm{hrs}$ & $1.01(0.77-1.31)$ \\
\hline $\begin{array}{l}\text { Schumer et al } \\
1976\end{array}$ & MP & $30 \mathrm{mg} / \mathrm{kg}$ & 1 or 2 doses & $0.3(0.13-0.72)$ \\
\hline $\begin{array}{l}\text { Klastersky et al } \\
1971\end{array}$ & $\mathrm{BM}$ & $1 \mathrm{mg} / \mathrm{kg}$ & $72 \mathrm{hrs}$ & $0.97(0.65-1.45)$ \\
\hline $\begin{array}{l}\text { Cooperative study } \\
\text { group } 1963\end{array}$ & $\mathrm{HC}$ & $\begin{array}{c}\text { 300mg followed } \\
\text { by } 50 \mathrm{mg} / \text { day }\end{array}$ & 6 days & $1.72(1.23-2.4)$ \\
\hline
\end{tabular}

$\mathrm{MP}=$ Methylprednisolone, $\mathrm{BM}=$ betamethasone, $\mathrm{HC}=$ hydrocortisone, $\mathrm{DM}=$ dexamethasone 


\section{Randomized Controlled Trials (RCT) conducted on low-dose or stress dose corticosteroids in sepsis and septic shock}

Two small double-blinded, randomized control trials demonstrated that prolonged treatment with low-dose hydrocortisone in septic shock reduced the time of shock reversal. ${ }^{29,30}$ The one published in 1998 showed that the administration of relatively low doses of hydrocortisone (100mg IV 8hourly) for 5 days to patients requiring catecholamines increased the speed and likelihood of shock reversal.

Again in 2005, a single center randomized trial also demonstrated that hydrocortisone induced shock reversal was greater in nonresponders than in responders of ACTH. ${ }^{31}$ The momentum in the reinforcement of LDC treatment for severe sepsis and septic shock was gradually gaining, until the results of the CORTICUS study were published. ${ }^{32}$

The CORTICUS trial was a multi-center, randomized, double blinded, placebo-controlled trial organized in 52 ICUs in nine countries.. The investigators acknowledged the increased use of LDC for septic patients because of the 2004 guidelines of the SSC. However, they also criticized the fact that the beneficial outcomes with steroids had only been noticed in septic shock patients who were non-responders in corticotropin tests and who remained hypotensive after aggressive fluid replacement.In this study 251 septic shock patients were given $50 \mathrm{mg}$ of IV hydrocortisone and 248 septic shock patients were treated with placebo every 6 hours for 5 days. ${ }^{32}$ At the time of enrollment, all patients had a corticotropin stimulation test, and $46.7 \%$ were identified as non-responders. No significant survival benefit was noticed in septic shock patients treated with corticosteroids and those receiving placebo (39.2\% vs. $36.1 \%$ mortality $\mathrm{P}=0$. 69).. Rapid and earlier shock reversal were more noticeable in septic shock patients treated with LDC than in the placebo group regardless of adrenal function $(\mathrm{P}=0.01)$. However, the extent of patients with insufficient shock reversal was comparable between the two groups. An ideal time for shock reversal was 3.3 days in the LDC group and 5.8 days in the placebo group.

An increased risk of superinfection and evidence of new sepsis or septic shock was noticed in the LDC-group (combined odds ratio, 1.37; 95\% CI, 1.05-1.79).
Although hydrocortisone therapy failed to improve 28day mortality in the CORTICUS study for all patients or non-responders and responders to ACTH, it also showed to improve organ dysfunction, which was reflected by a faster decrease in sequential organ failure assessment (SOFA) scores. ${ }^{33}$ There was a decline in the score of hydrocortisone-treated patients from day 0 to day 7 compared to the placebo-treated patients $(\mathrm{p}=0$. 0027), along with an improvement in cardiovascular organ dysfunction $(p=0.0005)$ and in liver failure $(p<0.0001)$ in the hydrocortisone-treated patients

Although the SSC recommendation was re-assessed in 2012, the optimum duration of therapy with LDCs in septic shock remained uncertain. Additionally, the patients in the early therapy group (corticosteroid initiated within 6 hours of onset of septic shock) had a $37 \%$ lower mortality rate than the late therapy group (corticosteroids after $6 \mathrm{hrs}$ of the onset of septic shock) (32\% vs. 51\%, $\mathrm{P}=0.01320$ ).

Finally, the authors concluded that the early initiation of a low-dose of corticosteroid therapy was associated with decreased mortality. ${ }^{34}$

However, low-dose steroids might benefit patients with the highest severity of illness.

\section{Systematic review and meta-analyses conducted on the use of corticosteroid in sepsis}

The studies published after 1997 started the low doses of glucocorticoids as late as 72 hours after the initiation of vasopressors, and continued for a minimum of 5 days followed by tapering over 5 to 7 days. On the contrary, studies before 1989 administered short courses of high doses of glucocorticoids earlier in the patients' septic episode. However, a regression analysis performed on all the studies showed that the relative survival benefit decreased linearly $(p=0.02)$ with the increasing dose of steroid, suggesting that low-dose steroids were effective, but subsequently became harmful with higher doses. The review concluded that short courses of highdose glucocorticoids could not show any significant survival benefit in sepsis. The review concluded that a short course of high-dose corticosteroids was associated with decreased survival in sepsis, whereas a 5-7 day's physiologic dose of hydrocortisone with subsequent tapering demonstrated improvement in survival and shock reversal in vasopressor-dependent septic shock. ${ }^{35}$

The other meta-analysis in the same year included 16 trials $(n=2063)$. The review concluded that in patients 
with severe sepsis and septic shock corticosteroids were not better than standard therapy for reducing all-cause mortality. Administration of corticosteroids had reversed shock without inducing side effects. However, long courses of LDCs reduced all-cause, intensive care, and in-hospital mortality at 28 days.

The role of low-dose steroids had been evaluated by Bayesian methodology in 2008. The study concluded that the strength of evidence (statistical and clinical) was weak for low-dose steroids and couldn't show any beneficial role for it. ${ }^{36}$

Another meta-analysis evaluated a number of smaller studies and stated that steroid therapy significantly reduced the incidence of vasopressor-dependent shock, but could not show any effect on mortality (RR, 1.00; 95\% CI, 0.84-1.18). ${ }^{37}$

In 2009, another meta-analysis of 21 studies was conducted, which was an update of previous analysis examining the effects of steroids during sepsis. Variable effects of steroids on mortality were found among the trials.

A small survival benefit was found in the trials published after 1997 which involved longer courses of low-dose steroids. But reported evidence of publication bias for improved survival was noticed in LDC trials (those published after 1997). A significant inverse-linear relationship between disease severity and an OR of mortality was found in the analysis, indicating that in less severely ill patients, steroids may be harmful. ${ }^{38}$ Another large meta-analysis in the same year found that when a group of RCTs published between 1998 and 2009 evaluating long duration of low-dose steroid therapy, were assessed in isolation, an improved mortality was observed in the treatment group compared to the control group $(37.5 \%$ vs $44.1 \%)$ (RR $0.84 ; 95 \% \mathrm{CI}$ 0.72 to $0.97 ; \mathrm{P}=0.02) .{ }^{39}$ There was also evidence of increased rates of hyperglycemia and hypernatremia with corticosteroid treatment in this study.

Efficacy of corticosteroid compared with control for hospital mortality, the extent of patients encountering shock resolution and also infective and non-infective complications were assessed by utilizing a Bayesian random-effects model and expressed as OR, $(95 \% \mathrm{CI})$. In this study 14, RCTs were identified, and Bayesian outcome probabilities were calculated as the probability (P) that $\mathrm{OR} \geq 1$. High-dose $(>1000 \mathrm{mg} /$ day hydrocortisone) corticosteroid trial was associated with a null $(\mathrm{n}=5$; OR0.91 $(0.31-1.25)$ or increased $(\mathrm{n}=4$, OR1.46 (0.73-2.16)) mortality probability ( $\mathrm{P}=42 \%$ and $89.3 \%$ respectively). Low-dose trials $(<1000 \mathrm{mg}$ /day $)$ were associated with a decreased mortality probability (20.4\% and 5.8\% respectively). In the low-dose trials, OR for shock resolution was increased. The patient's response to corticotropin stimulation was nondeterminant. An increased likelihood of risk-related treatment adequacy was recognized by a metaregression in the low-dose trials. The odds of complications were not increased with corticosteroids. ${ }^{40}$

The sepsis subcommittee of the American Academy of Emergency Medicine Clinical Practice Committee performed an extensive search of the contemporary literature and identified seven trials to evaluate the efficacy of LDC in the septic shock patients. Six of the seven trials reported the mortality outcome of septic shock patients.

Analysis of the data showed that the RR of 28 days allcause mortality in septic shock patients receiving LDCs was 0.92 (95\% CI 0.79-1.07). All the trials recorded data was concerning the shocking reversal or the rescission of vasopressors. Finally, the analysis revealed that an RR of shock reversal was 1.17 , which suggested that there was an improvement in shock reversal after corticosteroid therapy. However, the definition of reversal of shock was heterogeneous throughout the literature. ${ }^{41}$

The role of low-dose hydrocortisone in lessening septic shock and reducing short-term mortality was still unclear. Another meta-analysis of previous studies had been conducted to determine whether hydrocortisone could alleviate the effects of septic shock at 7 and 28 days and reduce the 28 -day mortality. 8 publications were incorporated in this meta-analysis. Low-dose hydrocortisone could not reduce the 28-day mortality $\left(\mathrm{n}=1063, \mathrm{OR}=0.891,95 \% \mathrm{CI}(0.69-1.15) .{ }^{42}\right.$ Cumulative meta-analysis showed that the OR value of 7-days shock reversal gradually decreased from 8.04 to 2.08 and $95 \%$ CI decreased from (1.94-33.30) to (1.58-2.73). On the other hand, a cumulative meta-analysis of 28-day shock reversal showed that the OR value decreased from 3.67 to 1.49 , and that about $95 \%$ CI decreased from (1.0113.04) to (1.12-1.99) as a function of the publication year. In both of the analysis, the OR values and $95 \% \mathrm{CI}$ 
were significantly higher than one indicating that despite the positive results of 7-days or 28-days shock reversal gradually weakened over the years, the findings were still significantly positive and became stable in recent years. ${ }^{42}$ In contrast with the previous meta-analyses, another recent systematic review with meta-analysis and trial sequential analysis (TSA) concluded that evidence to support or negate the use of steroids in septic patients is still lacking. A total of 35 trials randomizing 4682 patients were surveyed and investigated in the study. All but two trials had a risk of bias. No statistically significant results were diagnosed for any dose of steroid versus placebo or no intervention on mortality at maximal follow-up [RR 0.89; TSA adjusted (CI) 0.741.08]. No statistically significant effects were noticed on serious adverse effects other than mortality. Finally, TSA suggested that many more randomized patients are needed to draw a conclusion. ${ }^{43}$

Although in the CORTICUS trial shock reversal with the LDCs was achieved quickly, there was increased evidence of new sepsis or septic shock in the hydrocortisone group (OR 1.37, $\mathrm{p}<0.05) .{ }^{32}$ They also showed increased rates of hyperglycemia and hypernatremia in the treatment group. The study conducted by Oppert and colleagues did not show any increase in secondary infection, but there was a tendency for higher insulin requirement in the corticosteroidstreated patients. ${ }^{31}$

Persistent increase in the risk of new infections, GI bleeding and hyperglycemia was also observed in the Bayesian analysis. These findings clearly suggest that clinicians should not use hydrocortisone as a general adjuvant therapy in septic shock refractory to fluid and vasopressors therapies. The PROGRESS (Promoting Global Research Excellence in Severe Sepsis) registry enrolled 12,570 patients with severe sepsis to evaluate the global use of vasopressors and LDC at any time in intensive care units for the treatment of severe sepsis. ${ }^{44}$ They documented that around $80 \%$ patients were given vasopressors therapy and around 35\% received LDCs. It was also notified that $14 \%$ of severe sepsis patients, who did not even required vasopressors support, received corticosteroid therapy. Thus, it is likely that this inappropriate use of LDCs in the treatment of severe sepsis or septic shock is a result of the low cost of steroids and also due to the physician's belief in the potential safety and efficacy of this therapeutic strategy.
However, future trials investigating the role of steroids in sepsis and septic shock are ongong.

\section{Conclusion}

By reviewing the argument and presenting the findings of the papers it is clear that short courses of high-dose corticosteroids have no effect on outcomes rather it is harmful-most probably due to immunosuppression and re-infections.

Low-dose hydrocortisone for 5-7 days is a replacement therapy showed to improve outcomes. Numerous RCTs have demonstrated its beneficial effect on systemic blood pressure and on reducing the duration of vasopressor therapy. However, with the contradictory results from the recent large multicenter clinical trials the ability of corticosteroids to improve mortality in septic shock is still under a gray area. The optimum low dose steroid replacement strategy and length of treatment are still uncertain despite the standardized SSC guidelines.

\section{Conflict of interest: None}

\section{References}

1. Singer M, Deutschman C, Seymour C, Shankar-Hari M, Annane D, Bauer $M$ et al. Third International Consensus Definitions for Sepsis and Septic Shock (Sepsis-3). JAMA 2016;315(8):801.

2. Cepinskas G, Wilson J. Inflammatory Response in Microvascular Endothelium in Sepsis: Role of Oxidants. J Clin Biochem Nutr 2008;42(3):175-84.

3. De Backer D, Donadello K, Sakr Y, Ospina-Tascon G, Salgado D, Scolletta S et al. Microcirculatory Alterations in Patients With Severe Sepsis. Critical Care Medicine 2013;41(3): 791-99.

4. Snowden C, Kirkman E. The pathophysiology of sepsis. Continuing Education in Anaesthesia, Critical Care \& Pain 2002;2(1):11-14.

5. Prigent H, Maxime V, Annane D. Clinical review: corticotherapy in sepsis. Critical care (London, England) 2004;8(2):122-29.

6. Peeters B, Boonen E, Langouche L, Van den Berghe G. The HPA axis response to critical illness: New study results with diagnostic and therapeutic implications. Molecular and Cellular Endocrinology 2015;408:235-40.

7. Boonen E, Vervenne H, Meersseman P, Andrew R, Mortier L, Declercq P et al. Reduced Cortisol Metabolism during Critical Illness. New Eng J Med 2013;368(16):1477-88.

8. Cooper MS, Stewart PM. Corticosteroid Insufficiency in Acutely Ill Patients. New Eng J Med 2003;348:727-34.

9. Boonen E, Van Den BG. New concepts to further unravel adrenal insufficiency during critical illness. Eur J Endocrinol 2016; 175(1): R1-3. 
10. Peng J, Du B. Sepsis-related stress response: known knowns, known unknowns, and unknown unknowns. Critical Care 2010;14(4):179.

11. Marik P. Critical Illness-Related Corticosteroid Insufficiency. Chest 2009;135 (1):181-93.

12. Marik P, Pastores S, Annane D, Meduri G, Sprung C, Arlt, W et al. Recommendations for the diagnosis and management of corticosteroid insufficiency in critically ill adult patients: Consensus statements from an international task force by the American College of Critical Care Medicine. Critical Care Medicine 2008;36(6):1937-49.

13. Annane D. Adrenal Insufficiency in Sepsis. CPD 2008;14(19):1882-86.

14. Salgado DR, Verdeal JCR, Rocco JR. Adrenal function testing in patients with septic shock. Critical Care (London, England) 2006:10(5):R149.

15. Dellinger RP, Levy MM, Carlet JM, Bion J, Parker MM, Jaeschke R et al. Surviving Sepsis Campaign: International guidelines for management of severe sepsis and septic shock: 2008. Intensive Care Medicine 2008:3. Available from http:/ /doi.org/10.1007/s00134-007-0934-2.

16. Dellinger R, Levy M, Rhodes A, Annane D, Gerlach H, Opal $\mathrm{S}$ et al. Surviving Sepsis Campaign: International Guidelines for Management of Severe Sepsis and Septic Shock, 2012.Intensive Care Med 2013;39(2):165-228.

17. Annane D. Corticosteroids for septic shock. Journal Fur Anasthesie Und Intensivbehandlung 2003;10(1):7-8.

18. Annane D. The Role of ACTH and Corticosteroids for Sepsis and Septic Shock: An Update. Frontiers in Endocrinology 2016;7: 70 .

19. Hotchkiss R, Karl I. The Pathophysiology and Treatment of Sepsis. New Eng J Med 2003;348(2):138-50.

20. Goodwin JE. Endothelial glucocorticoid receptor is required for protection against sepsis. Proceedings of the National Academy of Sciences of the United States of America 2013, 110(1):306-11.

21. Marik PE, Zaloga GP. Adrenal insufficiency in the critically ill: a new look at an old problem. Chest 2002;122 (5):178496.

22. Strehl C, Buttgereit F. Molecular and Cellular Endocrinology Optimized glucocorticoid therapy/ : Teaching old drugs new tricks. Molecular and Cellular Endocrinology 2013;380(12):32-40.

23. Rhen T, Cidlowski J. Anti-inflammatory Action of Glucocorticoids - New Mechanisms for Old Drugs. New Eng J Med 2005; 353(16):1711-23.

24. van Leeuwen HJ, van der Bruggen T, van Asbeck BS, Boereboom FT. Effect of corticosteroids on nuclear factorkappaB activation and hemodynamics in late septic shock. Critical Care Medicine 2001;29(5):1074-77.
25. Casserly B, Gerlach H, Phillips GS, Lemeshow S, Marshall J C, Osborn TM et al. Low-dose steroids in adult septic shock: Results of the Surviving Sepsis Campaign. Intensive Care Medicine 2012;38(12):1946-54.

26. Dellinger R, Carlet J, Masur H, Gerlach H, Calandra T, Cohen $\mathrm{J}$ et al. Surviving Sepsis Campaign guidelines for management of severe sepsis and septic shock. Critical Care Medicine 2004;32(3):858-73.

27. Annane, Bellissant, Sebille, Lesieur, Mathieu, Raphael et al. Impaired pressor sensitivity to noradrenaline in septic shock patients with and without impaired adrenal function reserve. British Journal of Clinical Pharmacology 2002;46(6):589-97.

28. Contrael K, Killian A, Gregg S, Buchman T, Coopersmith C. Prescribing Patterns of Hydrocortisone in Septic Shock. Critical Care Medicine 2013;41(10):2310-17.

29. Bollaert P,charpentier D,Levy B,Debouveri M. Reversal of late septic shock with supraphysiologic doses of hydrocortisone. Critical Care Medicine 1998;26:645-50.

30. Briegel J,Forst H, Haller M,Schelling G, Kilger E, Kuprat G.Stress doses of hydrocortisone reverse hyperdynamic septic shock; a prospective,randomized, double blind, single centre study.Critical Care Medicine 1999;27:723-32.

31. Oppert M, Schindler R, Husung C, Offermann K, Gräf K, Boenisch $\mathrm{O}$ et al. Low-dose hydrocortisone improves shock reversal and reduces cytokine levels in early hyperdynamic septic shock. Critical Care Medicine 2005;33(11):2457-64.

32. Sprung C, Annane D, Keh D, Moreno R, Singer M, Freivogel $\mathrm{K}$ et al. Hydrocortisone Therapy for Patients with Septic Shock. New Eng J Med 2008;358(2):111-24.

33. Annane D. Corticosteroid Treatment and Intensive Insulin Therapy for Septic Shock in Adults. JAMA 2010;303(4):34148 .

34. Park H, Suh G, Song J, Yoo H, Jo I, Shin T et al. Early initiation of low-dose corticosteroid therapy in the management of septic shock: a retrospective observational study. Critical Care 2012;16(1):R3.

35. Minneci P. Meta-Analysis: The Effect of Steroids on Survival and Shock during Sepsis Depends on the Dose. Annals of Internal Medicine 2004;141(1):47.

36. Kalil A, Sun J. Why Are Clinicians Not Embracing the Results from Pivotal Clinical Trials in Severe Sepsis? A Bayesian Analysis. PLoS ONE 2008;3(5):e2291.

37. Sligl W, Milner Jr D, Sundar S, Mphatswe W, Majumdar S. Safety and Efficacy of Corticosteroids for the Treatment of Septic Shock: A Systematic Review and Meta Analysis. Clinical Infectious Diseases 2009;49(1):93-101.

38. Minneci P, Deans K, Eichacker P, Natanson C. The effects of steroids during sepsis depend on dose and severity of illness: an updated meta-analysis. Clinical Microbiology and Infection 2009;15(4):308-18.

39. Annane D, Bellissant E, Bollaert P, Briegel J, Confalonieri M, De Gaudio R et al. Corticosteroids in the Treatment of Severe Sepsis and Septic Shock in Adults. JAMA 2009;301 (22):2362. 
40. Moran JL, Graham PL, Rockliff S, Bersten AD. Updating the evidence for the role of corticosteroids in severe sepsis and septic shock: a Bayesian meta-analytic perspective. Critical Care (London, England) 2010;14(4):R134.

41. Sherwi, R, Garcia A, Bilkovski R. Do Low-dose Corticosteroids Improve Mortality or Shock Reversal in Patients with Septic Shock? A Systematic Review and Position Statement Prepared for the American Academy of Emergency Medicine. The Journal of Emergency Medicine 2012;43(1): 7-12.

42. Wang C, Sun J, Zheng J, Guo L, Ma H, Zhang Y et al. LowDose Hydrocortisone Therapy Attenuates Septic Shock in
Adult Patients but Does Not Reduce 28-Day Mortality. Anesthesia \& Analgesia 2014;118(2):346-57.

43. Volbeda M, Wetterslev J, Gluud C, Zijlstra J, van der Horst I et al. Glucocorticosteroids for sepsis: systematic review with meta-analysis and trial sequential analysis. Intensive Care Med 2015;41(7):1220-34

44. Beale R, Janes J, Brunkhorst F, Dobb G, Levy M, Martin G et al. Global utilization of low-dose corticosteroids in severe sepsis and septic shock: a report from the PROGRESS registry. Critical Care 2010;14(3):R102. 